\title{
Optimization of nano- and micro-iron transport through sand columns using polyelectrolyte mixtures
}

\author{
Bianca W. Hydutsky, Elizabeth J. Mack, Benjamin B. Beckerman, \\ Joanna M. Skluzacek, and Thomas E. Mallouk* \\ Department of Chemistry, The Pennsylvania State University, University Park, PA 16802 \\ Environmental Science and Technology, May 16, 2007 \\ Supporting Information: $11 \mathrm{pp}$.
}

Experimental details: Materials, iron particle transport experiments, particle characterization and instrumentation, calculations of eluted particle distributions.

Table S1. Mass balance analytical results for eluted and retained CIP-HQ iron.

Figure S1. X-ray powder diffraction (XRD) of CIP-HQ, -HF, and -OM.

Figure S2. XRD of RNIP stock solution.

Figure S-3. Fe 2p XPS spectrum of CIP-HQ and CIP-HF. 


\section{Materials}

Ottawa sand from VWR Scientific (CAS 7631-86-9) was dry sieved through a series of mesh screens to narrow the grain size dispersity for sand-packed columns. Sand that passed through a 40 mesh screen, but was unable to pass the 60 mesh screen, is hereafter referred to as 40 mesh sand. The 40 mesh sand was measured to be $160 \pm 45 \mu \mathrm{m}$ in diameter by optical microscopy. The Ottawa sand was analyzed by the Agricultural Analytical Services Laboratory at Penn State University and was found to contain $0.01 \%$ organic matter. Three carbonyl iron powders, CIP-OM, CIP-HF, and CIP-HQ were obtained from BASF. Nanocrystalline iron (Reactive Nano-Iron Particles, or RNIP) was provided by Toda America as a $25 \mathrm{wt} \%$ aqueous suspension. The RNIP samples were stored in sealed plastic containers, as received from the supplier, and were refrigerated at $1.7^{\circ} \mathrm{C}$ to prevent air oxidation and anaerobic corrosion. RNIP stock suspensions were made by diluting the received suspensions with water. The stock suspensions were used within 2-4 days of preparation.

Polyacrylic acid sodium salt (PAA), average MW 3500, was provided by Toda Kogyo, Ltd. Poly(sodium 4-styrenesulfonate) (PSS, $20 \%$ wt. in water, average MW 1,000,000) was purchased from Aldrich. A bentonite clay sample, provided by PARS Environmental, Inc., was analyzed at Agricultural Analytical Services Laboratory, Penn State University. This bentonite sample had a cation exchange capacity (CEC) of $22.1 \mathrm{meq} / 100 \mathrm{~g}$. The percent saturation of the CEC was $67.7 \% \mathrm{Ca}, 28.0 \% \mathrm{Mg}$, and $4.3 \% \mathrm{~K}$. The sample contained a high level of calcium (total calcium content $3400 \mathrm{ppm}$ ) suggesting the presence of a soluble calcium phase. Scanning electron microscopy (SEM) - Energy dispersive spectroscopy (EDS) confirmed the high calcium content. Therefore the CEC and percent saturations were calculated using a maximum 
exchangeable calcium level of $15 \mathrm{meq} / 100 \mathrm{~g}$. Textural analysis of the bentonite clay gave $75.1 \%$ clay, $9.5 \%$ silt, and $15.4 \%$ sand.

\section{Iron particle transport experiments}

Sample preparation. Iron particle suspensions, or stock suspensions, were made by mixing iron with water to the appropriate particle concentration. The appropriate polymer(s) or clay was then added to the iron particle suspension, followed by thorough mixing. From batch to batch, the concentration of iron in the suspensions varied $\pm 10 \%$; each sample was analyzed for iron prior to use, as described below, and the accuracy in the analysis was estimated to be $\pm 2 \%$. Nanopure water, resistivity of $18.2 \mathrm{M} \Omega-\mathrm{cm}$, was used to make all solutions/suspensions and to pack the columns used in transport experiments. All stock suspensions were shaken prior to injection into the columns.

Column preparation. One meter long glass columns (internal diameter $1.6 \mathrm{~cm}$ ) with a two-way valve at the lower end were used for column transport studies. First, a glass wool plug was pushed to the bottom of each column. The columns were filled with water and dry 40 mesh sand was then poured in to minimize trapping of air bubbles in the water-saturated sand. The length of the packed column is noted for each experiment. The porosity of the sand-packed columns was found to be $42 \%$ by comparing the mass of wet and dry columns. The specific gravity of Ottawa sand was measured as $2.63 \mathrm{~g} / \mathrm{cc}$. A slight pressure head was provided by a reservoir bulb at the top of the column. Flow rates were controlled by adjusting the two-way valve at the lower end of each column, and measuring the flow rate of water prior to the iron elution experiment. 
After rinsing the sand packed column with several pore volumes of water, $2.00 \mathrm{~mL}$ of CIP particle suspension $\left(5.0 \pm 0.5 \mathrm{mg} / \mathrm{mL}\right.$, or approximately $6 \times 10^{7}-5 \times 10^{8}$ particles $\left./ \mathrm{mL}\right)$ was introduced at the top of the column. The suspension was eluted in each case with deionized water. Particles not retained by the column were collected in $200 \mathrm{~mL}$ of eluted volume. All column tests were run at least in duplicate, and the iron content of each stock suspension and elution was quantified by a phenanthroline complexation method, described below. In experiments involving PAA/iron suspensions, the $\mathrm{pH}$ was controlled by the Na-PAA, which acts as a buffer. The $\mathrm{pH}$ of the CIP/PAA suspensions was 5.5 before and after being passed through a sand packed column.

Iron analysis by phenanthroline complexation. The phenanthroline method was used to quantitate the iron in both stock suspensions and in the eluted fraction collected from the columns. In each measurement, $1.00 \mathrm{~mL}$ of eluent or $0.050 \mathrm{~mL}$ of stock suspension was added to $4.0 \mathrm{~mL}$ hydrochloric acid $(1.0 \mathrm{M})$ to dissolve the iron in the sample, followed by $1.0 \mathrm{~mL}$ hydroxylamine hydrochloride $(100 \mathrm{~g} / \mathrm{L})$ to ensure complete conversion to the $\mathrm{Fe}^{2+}$ oxidation state. To increase the $\mathrm{pH}, 7.0 \mathrm{~mL}$ sodium acetate $(100 \mathrm{~g} / \mathrm{L})$ was then added. Then, $7.0 \mathrm{~mL} \mathrm{1,10-}$ phenanthroline $(1.0 \mathrm{~g} / \mathrm{L})$ was added to form the red-orange iron tris-phenanthroline complex $\left[\mathrm{Fe}(\mathrm{phen})_{3}\right]^{2+}$. The resulting solution was centrifuged and the absorbance at $\lambda=508 \mathrm{~nm}$ was measured by UV-visible spectroscopy, following calibration with a standard $\left(\mathrm{FeSO}_{4} \cdot\left(\mathrm{NH}_{4}\right)_{2} \mathrm{SO}_{4} \cdot 6 \mathrm{H}_{2} \mathrm{O}\right)$ iron solution (1). A linear calibration curve was obtained between absorbance values of 0.2 and 1.0. The iron concentration in each eluted sample, corrected for dilution, was compared to the concentration of iron in the particle stock suspension to determine the fraction that eluted from the column and the amount of iron retained. 
Background iron correction. A $1.0 \mathrm{mg} / \mathrm{mL}$ aqueous solution of PAA $(2.0 \mathrm{~mL})$ was eluted with $200 \mathrm{~mL}$ water through sand-packed columns of various lengths, following the procedure for injection of an iron stock solution. The eluted solutions collected from each column were analyzed for iron by the phenanthroline method. The iron released from the column by the PAA solution was subtracted from elution measurements as a background correction.

Analysis by scanning electron microscopy (SEM). Stock suspensions and collected elutions were imaged by SEM. The sizes of individual iron particles in each image were measured, and the frequency of each particle size was used to determine the particle size distribution of each sample, as shown in Figure 1.

CIP transport as a function of polymer concentration and flow rate. To investigate the effects of PAA in CIP iron stock suspensions, four different concentrations of PAA, $0.5 \mathrm{mg} / \mathrm{mL}$, $1.0 \mathrm{mg} / \mathrm{mL}, 2.0 \mathrm{mg} / \mathrm{mL}$, and $5.0 \mathrm{mg} / \mathrm{mL}$ were added to $5.0 \pm 0.5 \mathrm{mg} / \mathrm{mL}$ of CIP iron $\left(6 \times 10^{7}-5\right.$ x $10^{8}$ particles $/ \mathrm{mL}$ ) in water. These stock suspensions were passed through $26 \pm 2 \mathrm{~cm}$ long sand packed columns at a flow rate of $18 \mathrm{~mL} / \mathrm{min}(0.152 \mathrm{~cm} / \mathrm{s})$. The amount of iron eluted from each column was determined by the phenanthroline method. Flow rate effects were studied by comparing the elution of CIP through $52 \pm 3 \mathrm{~cm}$ long sand packed columns at 5, 10, and 18 $\mathrm{mL} / \mathrm{min}$. The two-way valve at the bottom of the column was adjusted to set each flow rate. The CIP and PAA concentrations in the stock suspensions were held constant at $5.0 \pm 0.5 \mathrm{mg} / \mathrm{mL}$ and $1.0 \mathrm{mg} / \mathrm{mL}$, respectively. Each $200 \mathrm{~mL}$ elution and the stock suspension were analyzed by the phenanthroline method and by SEM.

Quantification of retained iron. To measure the iron particles retained in the packing material, a specially designed glass transport column was used. This column consisted of two 
cylindrical sections connected by a short piece of flexible tubing, allowing the top reservoir section to be removed from the lower packed region. Following the elution experiment, the top section of the column was removed. The lower packed section was inverted, and the packing material exited the column as a plug that maintained its cylindrical shape. The packing material was extruded across aluminum foil folded to separate the column packing into individual segments. Each segment was air dried, weighed, and analyzed for iron content by using the phenanthroline method. To account for the background levels of iron in the packing material, a control sample was also analyzed for iron.

Table S1. Mass balance analytical results for eluted and retained CIP-HQ iron.

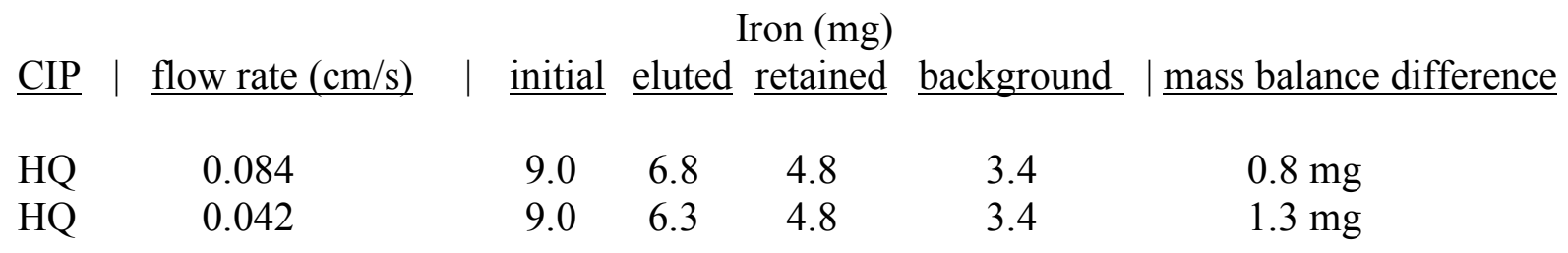

Transport of RNIP with polymer/clay mixtures. To study the effect of polyanion mixtures on iron nanoparticle filtration, different amounts of PAA, bentonite clay, and polystyrene sulfonate (PSS) were mixed with RNIP via a factorial design-of-experiment approach for three variables. Fifteen stock suspensions were made by mixing $10.0 \mathrm{mg} / \mathrm{mL}$ of RNIP with combinations of PAA, PSS and clay in water. The RNIP suspension was first diluted with water, and then the appropriate amount of solid PAA was added. PSS was added from a $20 \%$ solution, followed by the solid clay. Between each addition (PAA, PSS, clay), the suspension was agitated for $5 \mathrm{~min}$. The composition of each additive was determined by a central composite factorial design. For these experiments, the column lengths were $71 \pm 4 \mathrm{~cm}$ and the flow rate 
was held constant at $18 \mathrm{~mL} / \mathrm{min}$. The iron content in each stock suspension and elution was quantified by the phenanthroline method.

\section{Particle Characterization and Instrumentation}

Transmission electron microscope (TEM) images of nanoparticles were obtained using a JEOL 1200EXII microscope. Scanning electron microscope (SEM) images, taken with an FEI Quanta 200 ESEM, were used to determine the particle size distributions for the CIP samples. Iron particles in each image were individually measured and counted to determine the distribution of particle diameters in each sample. The diameter distribution of sand particles was determined by a similar procedure using optical microscope images from an Olympus SX60 microscope operated in bright field mode.

A Hewlett Packard 8452A diode array spectrophotometer was used for UV-visible analysis of iron-phenanthroline samples. X-ray powder diffraction (XRD) patterns were obtained with a Philips X'Pert MPD diffractometer, using monochromatized $\mathrm{Cu} \mathrm{K} \alpha_{1}(\lambda=$ $1.54053 \AA$ ) radiation. The electrokinetic properties of the carbonyl iron powder samples (CIPHQ, CIP-HF, and CIP-OM) in varying concentrations of PAA were measured using a ZetaPALS zeta potential analyzer (Brookhaven Instruments Corporation, Holtsville, NY). The iron powders were first suspended in the PAA solution and then allowed to settle before small aliquots were taken from the top of the supernatant. This procedure allows the largest particles to settle and gave reproducible mobility results. Electrokinetic analyses of iron powder control samples were also preformed using deionized water to suspend the particles. The electrophoretic mobility was measured at $25^{\circ} \mathrm{C}$, and the Smoluchowski model was used to convert the raw 
electrophoretic mobility data into zeta potential. Each reported result is the average of 5 replicate runs.

XRD analysis of CIP-HQ, -HF, and -OM (shown in Figure S1) suggests the CIP samples are composed of $\mathrm{Fe}^{0}$. In BASF HQ, a peak at 78.2 degrees is an artifact of the quartz sample holder. XRD of RNIP is shown in Figure S2. Although $\mathrm{Fe}^{0}$ is the major component of the RNIP sample, magnetite is also present. The manufacturer indicates that RNIP contains approximately $60 \% \mathrm{Fe}^{0}$. Using slightly aged samples, Lowry et al. measured 27 weight $\% \mathrm{Fe}^{0}$ (2).
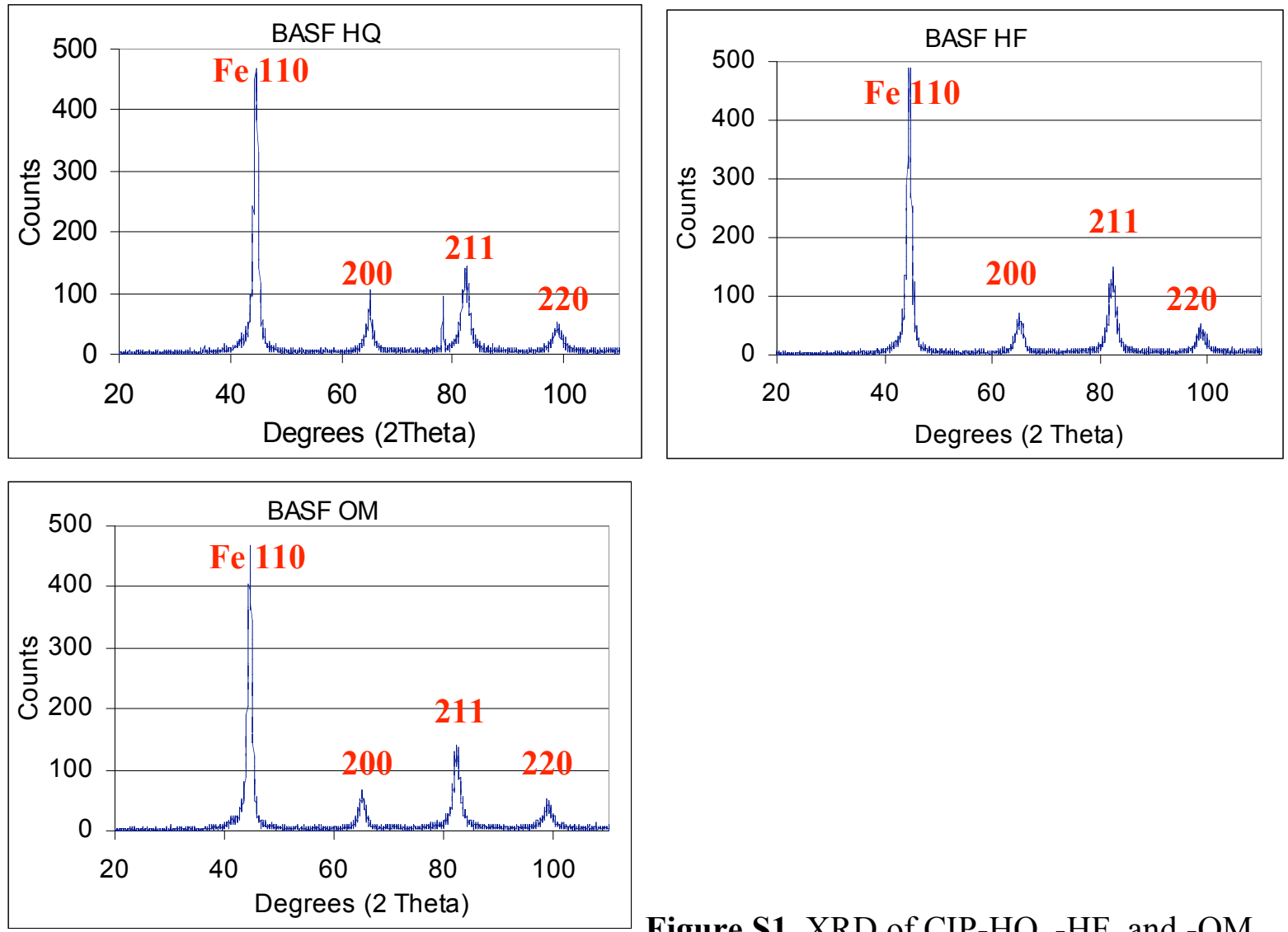

Figure S1. XRD of CIP-HQ, -HF, and -OM. 


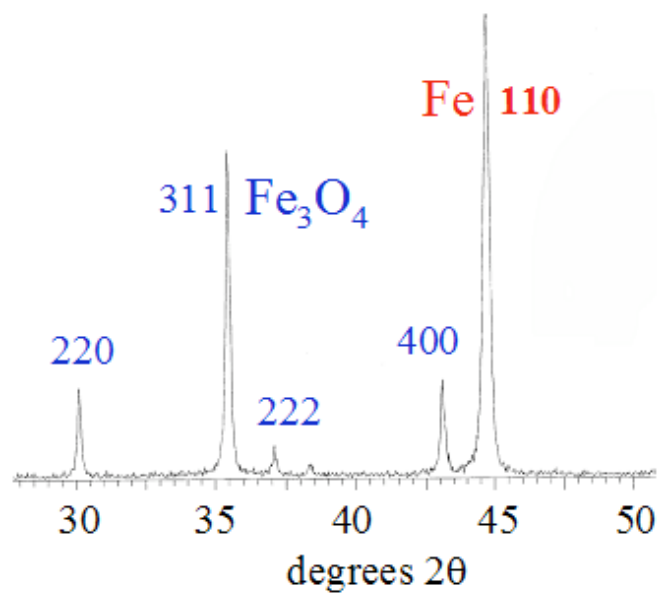

Figure S2. XRD of RNIP stock solution.

X-ray photoelectron spectra (XPS) of CIP-HQ and CIP-HF samples were obtained and are compared in Figure S3. The major peaks in the Fe $2 \mathrm{p}$ region of the spectrum are seen at 711 , 719 , and $725 \mathrm{eV}$, and are assigned respectively to $2 \mathrm{p}_{3 / 2}$, the $2 \mathrm{p}_{3 / 2}$ shake-up satellite, and $2 \mathrm{p}_{1 / 2}$, all corresponding to a thin surface oxide film (3). The smaller peak at $707 \mathrm{eV}$ binding energy is attributed to the $2 \mathrm{p} 3 / 2$ peak of $\mathrm{Fe}^{0}$ under the oxide film. The fact that this peak is visible in the spectra suggests that the oxide film is no more than 2-3 nm thick.

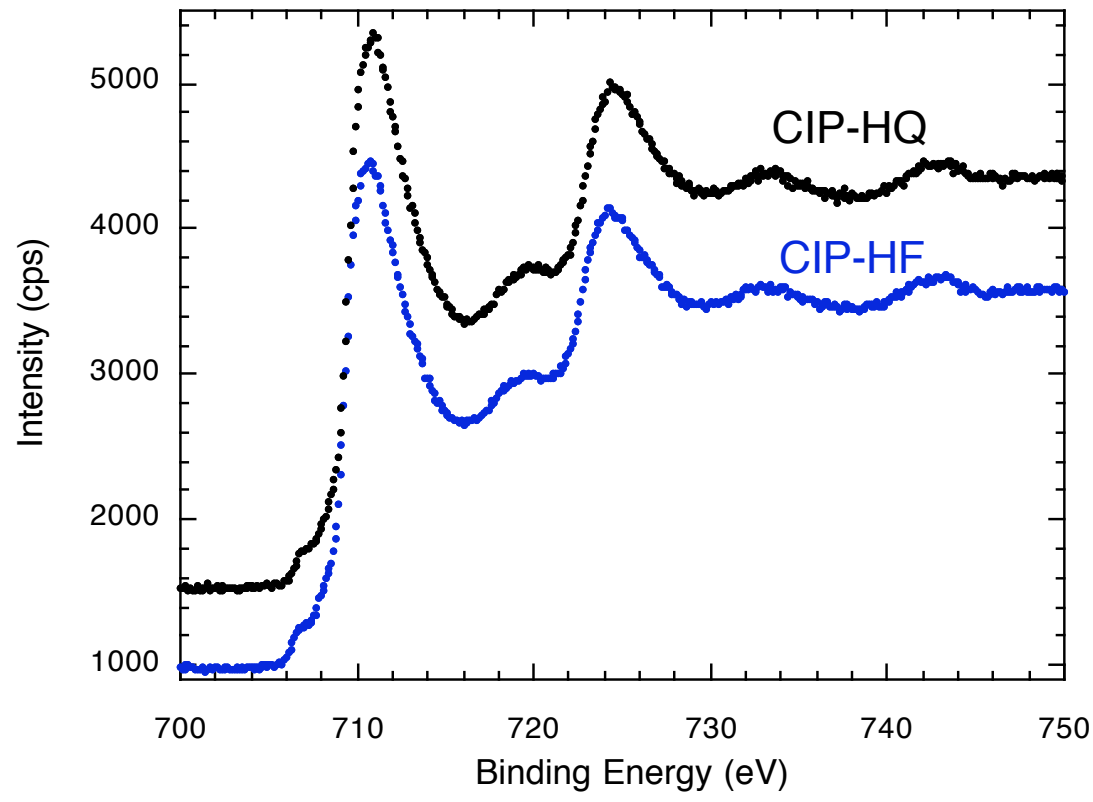

Figure S-3. Fe 2p XPS spectrum of CIP-HQ and CIP-HF. The CIP-HQ trace has been offset vertically for clarity. 


\section{Calculations of eluted particle size distributions}

In the Rajagopalan-Tien (or RT) model (Eqn. S1) (4), the three additive terms represent $\eta_{D}, \eta_{I}$,

$\eta=4.04 b_{H}{ }^{1 / 3} P e^{-2 / 3}+b_{H} L_{0}{ }^{1 / 8} R^{15 / 8}+0.00338 b_{H} S^{1 / 2} R^{-0.4}$

and $\eta_{G}$, respectively, and the parameters $\mathrm{b}_{\mathrm{H}}, \mathrm{Pe}, \mathrm{L}_{0}, \mathrm{R}^{*}$, and $\mathrm{S}^{*}$ can be calculated from the soil porosity, particle size, soil grain size, Darcy (or superficial) fluid velocity, particle density, and Hamaker constant.( ) A more recent model, which includes hydrodynamic and universal van der Waals interactions, has been developed by Tufenkji and Elimelech (TE) (5). In the TE equation (Eqn. S2), the three additive terms have the same meaning as in the RT equation, and the

$$
\eta=2.4 A_{S}^{1 / 3} N_{R}^{-0.081} N_{P e}{ }^{-0.715} N_{v d W}{ }^{0.052}+0.55 A_{S} N_{R}^{1.675} N_{A}^{0.125}+0.22 N_{R}^{-0.24} N_{G}^{1.11} N_{v d W}{ }^{0.053}
$$

dimensionless parameters $A_{S}, N_{R}, N_{P e}, N_{v d W}, N_{A}$, and $N_{G}$ can be calculated from the same set of physical parameters $(6)$.

\section{Literature Cited}

1. Skoog, D. A.; West, D. M. Analytical Chemistry, 4th ed.; Saunders College Publishing: Philadelphia, PA, 1986; pp 612-613.

2. Liu, Y.Q.; Majetich, S. A.; Tilton, R. D.; Sholl, D. S.; Lowry, G. V. TCE dechlorination rates, pathways, and efficiency of nanoscale iron particles with different properties. Environ. Sci. Technol. 2005, 39, 1338-1345.

3. Li, X.-Q.; Zhang, W.-X., Iron nanoparticles: the core-shell structure and unique properties for Ni(II) sequestration. Langmuir 2006, 22, 4638-4642. 
4. Rajagopalan, R.; Tien, C. Trajectory analysis of deep-bed filtration with the sphere-in-cell porous media model. AIChE J. 1976, 22, 523-533.

5. Tufenkji, N.; Elimelech, M. Correlation Equation for Predicting Single-Collector Efficiency in Physicochemical Filtration in Saturated Porous Media. Environ. Sci. Technol. 2004, 38, 529-536.

6. The porosity function $A_{S}$ is defined as $A_{S}=\left(\frac{2\left(1-\gamma^{5}\right)}{2-3 \gamma+3 \gamma^{5}-2 \gamma^{6}}\right)$, where $\gamma=(1-\theta)^{1 / 3}$. The Peclet number $N_{P e}=u d_{c} / D_{p}$, where $D_{p}=k_{B} T /\left(3 \pi \mu d_{p}\right)$. $u$ is the Darcy flow velocity, $k_{B}$ is Boltzmann's constant, $\mathrm{T}$ is the absolute temperature, and $\mu$ is the fluid viscosity. The other parameters are given by $\mathrm{N}_{\mathrm{vdW}}=\mathrm{A} / \mathrm{k}_{\mathrm{B}} \mathrm{T}, N_{A}=\frac{A}{3 \pi d_{p}{ }^{2} \mu u}, \mathrm{~N}_{\mathrm{R}}=\mathrm{d}_{\mathrm{p}} / \mathrm{d}_{\mathrm{c}}$, and $N_{G}=\frac{d_{p}{ }^{2} g \Delta \rho}{18 \mu u}$, where $\mathrm{A}$ is the particle Hamaker constant, $\mathrm{g}$ is the gravitational constant, $\Delta \rho$ is the difference in specific gravity between the particle and the fluid, and $d_{p}$ and $d_{c}$ are the particle and collector grain diameters, respectively. See (3) for complete details. For iron microparticle transport calculations, we used $\Theta=42 \%, \Delta \rho=6 \mathrm{~g} / \mathrm{cm}^{3}, \mathrm{~A}=1 \times 10^{-20} \mathrm{~J}$, and T $=298 \mathrm{~K}$. 